\title{
BMJ Open Impact of interankle blood pressure difference on major adverse cardiovascular events in cryptogenic stroke patients without peripheral artery disease: a retrospective cohort study
}

\author{
Minho Han (D) , ${ }^{1,2}$ Minyoul Baik, ${ }^{1}$ Young Dae Kim, ${ }^{1,2}$ Junghye Choi, ${ }^{1}$ Kangsik Seo, ${ }^{1}$ \\ Eunjeong Park, ${ }^{2} \mathrm{Ji} \mathrm{Hoe} \mathrm{Heo,}{ }^{1,2}$ Hyo Suk Nam (D) 1,2
}

To cite: Han M, Baik M, Kim YD, et al. Impact of interankle blood pressure difference on major adverse cardiovascular events in cryptogenic stroke patients without peripheral artery disease: a retrospective cohort study. BMJ Open 2022;12:e054760. doi:10.1136/ bmjopen-2021-054760

- Prepublication history for this paper is available online. To view these files, please visit the journal online (http://dx.doi. org/10.1136/bmjopen-2021 054760).

Received 22 June 2021 Accepted 04 February 2022

Check for updates

(c) Author(s) (or their employer(s)) 2022. Re-use permitted under CC BY-NC. No commercial re-use. See rights and permissions. Published by BMJ.

${ }^{1}$ Department of Neurology, Yonsei University College of Medicine, Seoul, Korea ${ }^{2}$ Integrative Research Center for Cerebrovascular and Cardiovascular Diseases, Yonsei University College of Medicine, Seoul, Korea

Correspondence to

Dr Hyo Suk Nam;

hsnam@yuhs.ac

\section{ABSTRACT}

Objective We investigated whether interankle blood pressure difference (IAND) can predict major adverse cardiovascular events (MACEs) in patients with cryptogenic stroke (CS) without peripheral artery disease (PAD).

Design A retrospective cohort study.

Setting Retrospective medical record data of patients with first-ever acute cerebral infarction who were admitted between 1 January 2007 and 31 July 2013.

Participants CS patients admitted within 7 days of symptom onset were included.

Outcome measures MACEs were defined as stroke recurrence, myocardial infarction occurrence, or death. Survival analyses were conducted using the Kaplan-Meier method and Cox regression analysis.

Methods Consecutive CS patients without PAD who underwent ankle-brachial index (ABI) measurements were enrolled. PAD was defined if a patient had an $A B I$ of $<0.90$ or a history of angiographically confirmed PAD. Systolic and diastolic IANDs were calculated as follows: right ankle blood pressure-left ankle blood pressure.

Results A total of 612 patients were enrolled and followed up for a median 2.6 (interquartile range, 1.0-4.3) years. In the Cox regression analysis, systolic and diastolic IANDs $\geq 15 \mathrm{~mm} \mathrm{Hg}$ were independently associated with MACEs in CS patients without PAD (hazard ratio (HR) 2.115, 95\% confidence interval (CI) 1.230 to 3.635 and HR 2.523, $95 \% \mathrm{Cl} 1.086$ to 5.863 , respectively). In the subgroup analysis, systolic IAND $\geq 15 \mathrm{~mm} \mathrm{Hg}$ was independently associated with MACEs in older patients (age $\geq 65$ years) (HR 2.242, 95\% Cl 1.170 to 4.298) but not in younger patients (age $<65$ years).

Conclusions Large IAND is independently associated with the long-term occurrence of MACEs in patients with CS without PAD. In particular, the association between IAND and MACEs is only valid in elderly patients.

\section{INTRODUCTION}

Despite extensive stroke evaluations, the cause of approximately 20\%-30\% cerebral infarction cannot be determined. This type of stroke is called cryptogenic stroke (CS).

\section{Strengths and limitations of this study}

This was a single-centre, hospital-based, retrospec tive observational study.

- We investigated the prognostic value of interankle blood pressure difference (IAND) in cryptogenic stroke patients without peripheral artery disease.

- We used a multivariate Cox proportional hazards regression to determine the independent association between IAND and major adverse cardiovascular events (stroke recurrence, myocardial infarction occurrence or all-cause death).

- Design permits only association rather than determination of causality.

Overt peripheral artery disease (PAD) is not frequent in patients with $\mathrm{CS}^{2}{ }^{2}$ Our previous study has shown that the interankle blood pressure difference (IAND) obtained during ankle-brachial index (ABI) measurements can identify mild PAD and predict poor shortterm and long-term outcomes in patients with ischaemic stroke without overt PAD. ${ }^{3}$ The prognosis of patients with CS is largely unknown because of concealed etiologies. In particular, age is a strong predictor of stroke recurrence and death in patients with $\mathrm{CS}^{4}$ and influences the likelihood of various etiologies. ${ }^{5}$ Therefore, we investigated the association between IAND and major adverse cardiovascular events (MACEs) in patients with CS without overt PAD. In addition, we evaluated whether the prognostic value of IAND differs according to age.

\section{MATERIALS AND METHODS}

Patients and evaluation

Our prospectively collected stroke database was retrospectively reviewed. The candidates 


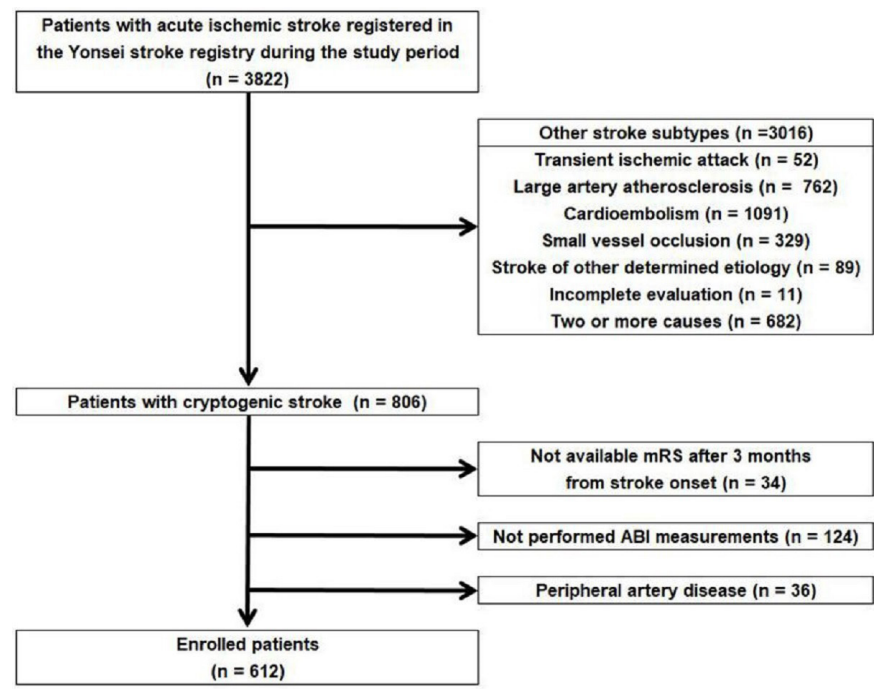

Figure 1 Flow chart of participants according to inclusion and exclusion criteria. ABI, ankle-brachial index; mRS, modified Rankin Scale.

were patients with first-ever acute cerebral infarction who were admitted within 7 days of symptom onset between 1 January 2007 and 31 July 2013 and who were registered in the Yonsei Stroke Registry. If symptom onset was unclear, the last known normal time was used. Acute cerebral infarction was defined as sudden onset of acute neurological deficits of presumed vascular aetiology lasting 24 hours or evidence of acute infarction on brain CT or MRI. During the study period, 3822 consecutive patients with acute ischaemic stroke were registered. The stroke subtypes were determined during weekly conferences and classified according to the Trial of ORG 10172 in Acute Stroke Treatment classification. ${ }^{6}$ We defined CS as stroke of an undetermined aetiology attributable to negative evaluation findings, despite extensive work-up. The exclusion criteria were stroke subtypes other than CS, including transient ischaemic attack $(\mathrm{n}=52)$, large artery atherosclerosis $(n=762)$, cardioembolism $(n=1007)$, small vessel occlusion $(n=329)$, stroke of other determined causes $(n=89)$, and stroke of two or more causes $(n=682)$; incomplete evaluation $(n=11)$; follow-up loss $(n=34)$; not performed bilateral ABI measurements $(n=124)$; and PAD $(n=36)$. After exclusion, a total of 612 patients with CS were finally enrolled in this study (figure 1).

\section{Patient and public involvement}

No patient was involved.

\section{Demographic characteristics and risk factors}

We collected data on the baseline characteristics, including gender, age and neurological deficit (National Institutes of Health Stroke Scale (NIHSS) score) at admission, presence of risk factors and laboratory data (total cholesterol, low-density lipoprotein, high-density lipoprotein, and triglyceride). ${ }^{3}$ Hypertension was defined as being present when a patient had been taking antihypertensive medications or had a systolic blood pressure (BP) of $\geq 140 \mathrm{~mm}$
$\mathrm{Hg}$ or a diastolic BP of $\geq 90 \mathrm{~mm} \mathrm{Hg}$ on repeated measurements during admission. Diabetes mellitus was diagnosed for fasting plasma glucose levels of $\geq 7.0 \mathrm{mmol} / \mathrm{L}$ or taking oral hypoglycaemic agent or insulin. Hypercholesterolaemia was diagnosed for low-density lipoprotein cholesterol levels of $\geq 4.1 \mathrm{mmol} / \mathrm{L}$, total cholesterol levels of $\geq 6.2 \mathrm{mmol} / \mathrm{L}$, or currently taking lipid-lowering agents. Congestive heart failure was determined from the history of heart failure diagnosis, treatment with loop diuretics, and ejection fraction of $\leq 35 \%$ on echocardiography. Coronary artery disease was diagnosed when a patient had a previous history of coronary artery disease (acute myocardial infarction, unstable angina, coronary artery bypass graft or percutaneous coronary artery stent/angioplasty) or the presence of significant stenosis $(\geq 50 \%)$ in any of three main coronary arteries on multislice CT coronary angiography taken during admission. Current smoking was defined as having smoked a cigarette within 1 year prior to admission. PAD was determined if a patient had an ABI of $<0.90$ or a history of angiographically confirmed PAD.

\section{Measurement of $\mathrm{ABI}$ and brachial-ankle pulse wave velocity}

$\mathrm{ABI}$ and brachial-ankle pulse wave velocity were measured in the supine position once at $<7$ days from admission using an automatic device (VP-1000; Colin, Komaki, Japan), which has been validated previously. ${ }^{7}$ This device simultaneously measures four-limb pulse wave forms and $\mathrm{BP}$ using the oscillometric method. ABI was calculated by the ratio of the ankle systolic BP divided by the higher systolic BP of the arms. Systolic and diastolic IANDs were extracted as BPs from both legs and calculated as Iright ankle BP - left ankle BPI. Brachial-ankle pulse wave velocity on each side was automatically calculated as the transmission distance divided by the transmission time and expressed in centimetres per second. Transmission distance from the arm to each ankle was automatically calculated according to the patient's height. Transmission time was defined as the time interval between the initial increase of brachial and tibial waveforms. The higher values of brachial-ankle pulse wave velocity on both sides were used for analysis.

\section{Follow-up and outcome measures}

After discharge, each patient was followed regularly for 3 months, 1 year, and every year thereafter. Every follow-up visit, medical information such as the occurrence of cardiovascular events, newly detected vascular risk factors, lifestyle modification after stroke and readmission to other hospitals were obtained through direct interviews with neurologists or through clinical research associates at the outpatient clinic. When the patients missed a scheduled visit, we obtained the information from the patients or their proxy through a telephone interview with a structured questionnaire. Short-term functional outcomes at 3 months were determined by a structured interview using the modified Rankin Scale. Poor functional outcome was defined as a modified Rankin Scale of $\geq 3$. Deaths among 
participants from 1 January 2007 to 31 December 2013 were confirmed by matching the information in the death records and identification numbers assigned to the participants at birth. We obtained data for the date and causes of death from the Korean National Statistical Office, which were identified based on death certificates. MACEs were defined as any stroke recurrence, myocardial infarction occurrence or all-cause death. The censoring date was 31 December 2013.

\section{Statistical analysis}

SPSS for Windows (V.25, SPSS) was used for the statistical analysis. Cut-off values for systolic and diastolic IANDs were based on those used in the previous study, which reported that IAND of $\geq 15 \mathrm{~mm} \mathrm{Hg}$ was a cut-off value that could predict mortality in stroke patients and elderly people. ${ }^{38}$ Patients with CS were divided into two groups according to the presence of IAND $\geq 15 \mathrm{~mm} \mathrm{Hg}$. The statistical significance of intergroup differences was assessed using the $\chi^{2}$ or Fisher's exact test for categorical variables and independent two-sample t-test or Mann-Whitney $\mathrm{U}$ test for continuous variables. Data were expressed as mean \pm standard deviation or median (interquartile range) for continuous variables and number (\%) for categorical variables. Survival curves were generated according to the Kaplan-Meier method and compared using the log-rank test. Multivariable Cox proportional hazards regression was performed to determine the independent association between IAND and MACEs. We adjusted gender, age, NIHSS score at admission, cardiovascular risk factors (hypertension, diabetes mellitus, hypercholesterolaemia, current smoking, congestive heart failure, and coronary artery disease), and variables (brachial-ankle pulse wave velocity and high-density lipoprotein) that exhibited a $\mathrm{p}<0.05$ in the univariable analysis. Subgroup analysis was performed with Cox regression analysis based on $\geq 65$ years or $<65$ years old. All $p$ values were two tailed, and differences were considered significant at $\mathrm{p}<0.05$.

\section{RESULTS}

\section{Prevalence of PAD}

The prevalence of PAD was significantly different depending on the stroke subtype. The prevalence of PAD was $5.6 \%$ in CS, $13.0 \%$ in large artery atherosclerosis,

Table 1 Prevalence of peripheral artery disease among ischaemic stroke subtypes

\begin{tabular}{lll}
\hline & \multicolumn{2}{l}{ Peripheral artery disease } \\
\cline { 2 - 3 } & Number (\%) & P value \\
\hline $\begin{array}{l}\text { Cryptogenic stroke }(n=682) \\
\text { Large artery atherosclerosis } \\
(n=615)\end{array}$ & $80(13.6)$ & $<0.001$ \\
Cardioembolism $(n=776)$ & $63(8.1)$ & \\
Small vessel occlusion $(n=281)$ & $16(5.7)$ & \\
\hline
\end{tabular}

$8.1 \%$ in cardioembolism, and $5.7 \%$ in small vessel occlusion (table 1).

\section{Clinical characteristics}

The mean patient age was $64.7 \pm 12.5$ years. Of the total patients with CS, $60.1 \%$ were men and $17.2 \%$ had a poor functional outcome. Systolic and diastolic IANDs $\geq 15 \mathrm{~mm}$ $\mathrm{Hg}$ were noted in $8.5 \%$ and $2.3 \%$ patients, respectively. Compared with patients with systolic IAND $<15 \mathrm{~mm} \mathrm{Hg}$, those with systolic IAND $\geq 15 \mathrm{~mm} \mathrm{Hg}$ were older, had lower high-density lipoprotein, had higher brachial-ankle pulse wave velocity and systolic and diastolic IANDs (all $\mathrm{p}<0.05)$. They also showed a poor functional outcome at 3 months $(p<0.001)$. Patients with diastolic IAND $\geq 15 \mathrm{~mm}$ $\mathrm{Hg}$ had higher systolic and diastolic IANDs than those with diastolic IAND $<15 \mathrm{~mm} \mathrm{Hg}$ (all $\mathrm{p}<0.05$ ) (table 2). Large IAND was significantly associated with lower ABI in both systolic and diastolic (all $\mathrm{p}<0.05)$ (table 3 ).

\section{Association between IAND and MACE}

All patients were followed up for a median of 2.6 (interquartile range, 1.0-4.3) years. The incidence of MACEs was $21.2 \%$. In the Kaplan-Meier survival analyses, the incidence of MACEs was higher in patients with systolic and diastolic IANDs $\geq 15 \mathrm{~mm} \mathrm{Hg}$ than in those with systolic and diastolic IANDs $<15 \mathrm{~mm} \mathrm{Hg}$ (log-rank test, all $\mathrm{p}<0.05$ ) (figure 2). In the multivariable Cox regression analysis, systolic and diastolic IANDs $\geq 15 \mathrm{~mm} \mathrm{Hg}$ were independent predictors of MACEs in patients with CS (hazard ratio (HR) $2.115,95 \%$ confidence interval (CI) 1.230 to 3.635 and HR: $2.523,95 \%$ CI 1.086 to 5.863 , respectively). In the subgroup analysis according to age, systolic IAND $\geq 15 \mathrm{~mm} \mathrm{Hg}$ in elderly patients (age: $\geq 65$ years) was significantly associated with MACEs (HR 2.242, 95\% CI 1.170 to 4.298 ), but not diastolic IAND $\geq 15 \mathrm{~mm} \mathrm{Hg}$. In younger patients (age: $<65$ years), there was no significant association between IAND and MACEs (table 4).

\section{DISCUSSION}

This study showed that systolic and diastolic IANDs were associated with MACEs in patients with CS without overt $\mathrm{PAD}$, after adjusting for well-known prognosis predictors. In particular, large IAND was associated with outcomes in elderly patients with CS. These findings suggest that large IAND may be a useful predictor for the long-term occurrence of MACEs in elderly patients with CS without overt PAD.

Interarm BP difference has been associated with recurrent stroke, ${ }^{9}$ poor prognosis, ${ }^{10}$ and mortality. ${ }^{11}$ Similarly, previous studies have been shown that large IAND is associated with all-cause and cardiovascular mortality in the elderly population and patients with acute myocardial infarction. ${ }^{8} 12$ Our previous study also reported that large IAND could predict poor short-term and long-term outcomes in patients with acute ischaemic stroke without $\mathrm{PAD}$ and that the prognostic value of IAND was superior to the interarm BP difference. ${ }^{3}$ However, the prognostic 
Table 2 Clinical characteristics and comparison of study patients with and without interankle blood pressure difference $\geq 15 \mathrm{~mm} \mathrm{Hg}$

\begin{tabular}{|c|c|c|c|c|c|c|c|}
\hline & $\begin{array}{l}\text { Total } \\
(n=612)\end{array}$ & $\begin{array}{l}\text { Systolic } \\
\text { IAND }<15 \mathrm{~mm} \\
\mathrm{Hg} \\
(\mathrm{n}=560)\end{array}$ & $\begin{array}{l}\text { Systolic } \\
\text { IAND } \geq 15 \mathrm{~mm} \\
\mathrm{Hg} \\
(\mathrm{n}=52)\end{array}$ & $P$ value & $\begin{array}{l}\text { Diastolic } \\
\text { IAND }<15 \mathrm{~mm} \\
\mathrm{Hg} \\
(\mathrm{n}=598)\end{array}$ & $\begin{array}{l}\text { Diastolic } \\
\text { IAND } \geq 15 \mathrm{~mm} \\
\mathrm{Hg} \\
(\mathrm{n}=14)\end{array}$ & $P$ value \\
\hline Men & $368(60.1)$ & $332(59.3)$ & $36(69.2)$ & 0.161 & $360(60.2)$ & $8(57.1)$ & 0.817 \\
\hline NIHSS score at admission & $2.0(1.0,5.0)$ & $2.0(1.0,4.0)$ & $3.0(1.0,7.0)$ & 0.239 & $2.0(1.0,4.0)$ & $3.0(2.0,5.8)$ & 0.456 \\
\hline $\begin{array}{l}\text { Brachial-ankle pulse wave } \\
\text { velocity, } \mathrm{cm} / \mathrm{s}\end{array}$ & $2050.0 \pm 534.4$ & $2035.0 \pm 534.2$ & $2195.5 \pm 510.5$ & 0.048 & $2039.1 \pm 524.1$ & $2476.6 \pm 779.6$ & 0.079 \\
\hline \multicolumn{8}{|l|}{ Risk factors } \\
\hline Hypertension & $466(76.1)$ & $427(76.3)$ & $39(75.0)$ & 0.840 & $454(75.9)$ & $12(85.7)$ & 0.536 \\
\hline Congestive heart failure & $10(1.6)$ & $10(1.8)$ & $0(0.0)$ & 1.000 & $10(1.7)$ & $0(0.0)$ & 1.000 \\
\hline Coronary artery disease & $109(17.8)$ & $102(18.2)$ & $7(13.5)$ & 0.392 & $105(17.6)$ & $4(28.6)$ & 0.289 \\
\hline \multicolumn{8}{|l|}{ Laboratory findings } \\
\hline Total cholesterol, mg/dL & $182.2 \pm 42.1$ & $182.3 \pm 42.2$ & $181.3 \pm 42.0$ & 0.865 & $182.4 \pm 42.3$ & $177.7 \pm 36.5$ & 0.685 \\
\hline LDL, mg/dL & $114.3 \pm 38.1$ & $113.9 \pm 38.2$ & $117.9 \pm 36.6$ & 0.471 & $114.2 \pm 38.3$ & $115.8 \pm 29.1$ & 0.881 \\
\hline $\mathrm{HDL}, \mathrm{mg} / \mathrm{dL}$ & $42.4 \pm 10.8$ & $42.8 \pm 10.7$ & $39.1 \pm 10.4$ & 0.018 & $42.5 \pm 10.7$ & $40.2 \pm 12.0$ & 0.496 \\
\hline Triglyceride, mg/dL & $128.9 \pm 89.9$ & $129.6 \pm 91.6$ & $121.7 \pm 69.0$ & 0.545 & $129.4 \pm 90.7$ & $108.7 \pm 43.3$ & 0.545 \\
\hline \multicolumn{8}{|l|}{ IAND, $\mathrm{mm} \mathrm{Hg}$} \\
\hline Systolic IAND & $6.71 \pm 5.88$ & $5.43 \pm 3.81$ & $20.42 \pm 6.74$ & $<0.001$ & $6.55 \pm 5.67$ & $13.36 \pm 10.04$ & 0.396 \\
\hline
\end{tabular}

Data are expressed as mean \pm standard deviation, median [interquartile range], or number (\%).

HDL, high-density lipoprotein; IAND, interankle blood pressure difference; LDL, low-density lipoprotein; NIHSS, National Institutes of Health Stroke Scale.

effects of IAND in stroke patients are not fully established. Particularly, little is known about the prognostic value of IAND according to stroke subtypes such as CS.

In the Oxford Vascular Study, patients with CS had the lowest frequency of PAD and a lower atherosclerotic burden than those with other stroke subtypes. ${ }^{2}{ }^{13}$ We also found that the prevalence of overt PAD was lower in

\begin{tabular}{lll}
\hline Table 3 Association between IAND and ABI & \\
\hline & \multicolumn{3}{c|}{ Mean ABI value } & \\
\cline { 2 - 3 } & Mean \pm SD & P value \\
\hline Systolic IAND & & \\
$<15 \mathrm{~mm} \mathrm{Hg}(n=560)$ & $1.139 \pm 0.073$ & 0.037 \\
$\geq 15 \mathrm{~mm} \mathrm{Hg}(\mathrm{n}=52)$ & $1.116 \pm 0.084$ & \\
Diastolic IAND & & \\
$<15 \mathrm{~mm} \mathrm{Hg}(\mathrm{n}=598)$ & $1.138 \pm 0.073$ & 0.016 \\
$\geq 15 \mathrm{~mm} \mathrm{Hg}(\mathrm{n}=14)$ & $1.090 \pm 0.085$ & \\
\hline
\end{tabular}

$\mathrm{ABI}$, ankle-brachial index; IAND, interankle blood pressure difference; SD, standard deviation. patients with CS compared to those with known etiologies. Nevertheless, intermittent claudication, the most common symptom of PAD, was reported to be quite frequent and associated with a worse health-related quality of life in patients with coronary heart disease without overt PAD. ${ }^{14}$ A recent study also found that $13.1 \%$ of stroke patients with normal $\mathrm{ABI}(0.9 \leq \mathrm{ABI} \leq 1.4)$ had $\mathrm{PAD}$, defined as low toe-brachial index. ${ }^{15}$ In this context, screening for mild PAD in patients with CS may provide additional information to estimate the prognosis. Therefore, we excluded
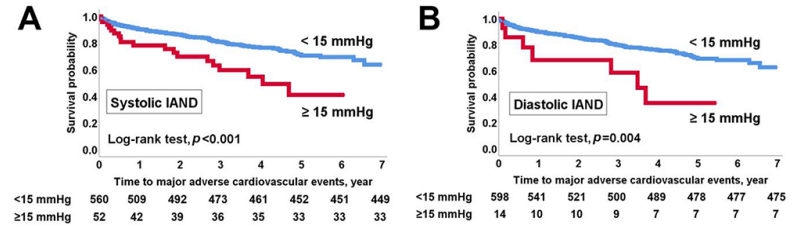

Figure 2 Kaplan-Meier survival analysis. (A) Major adverse cardiovascular events according to systolic IAND $\geq 15 \mathrm{~mm}$ $\mathrm{Hg}$; (B) major adverse cardiovascular events according to diastolic IAND $\geq 15 \mathrm{~mm} \mathrm{Hg}$. IAND, interankle blood pressure difference. 
Table 4 Cox regression analysis of major adverse cardiovascular events

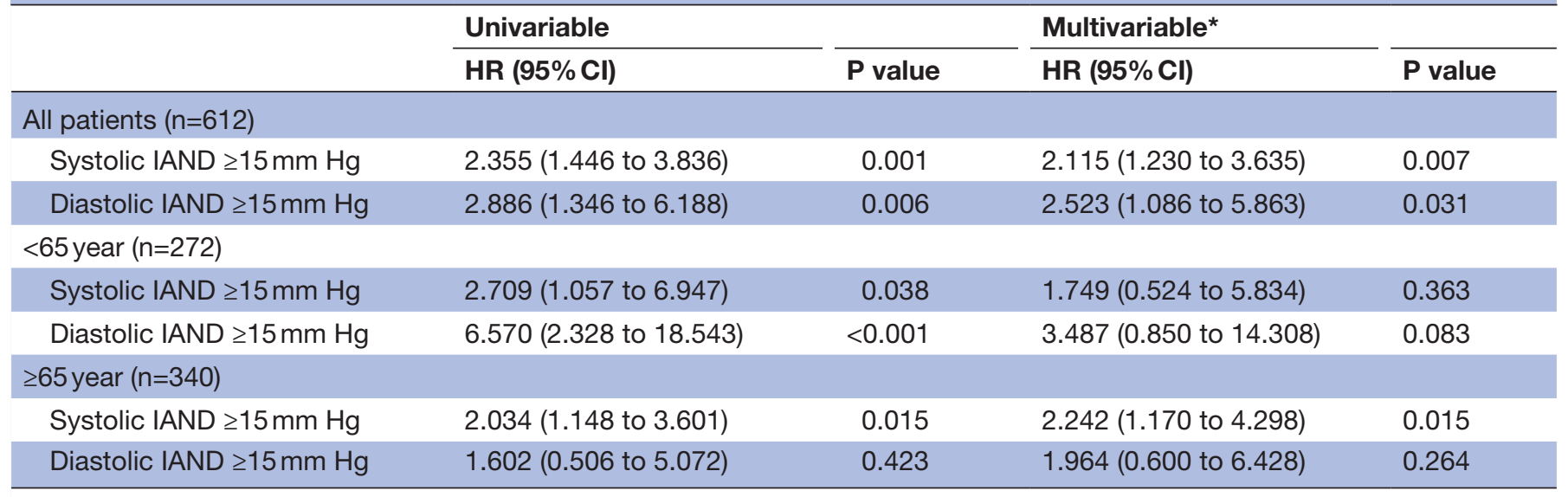

Data are derived from multivariable Cox proportional hazards regression analysis.

*Adjusted for gender, age, National Institutes of Health Stroke Scale score at admission, brachial-ankle pulse wave velocity, high-density lipoprotein, hypertension, diabetes mellitus, hypercholesterolaemia, current smoking, congestive heart failure, and coronary artery disease. $\mathrm{Cl}$, confidence interval; HR, hazard ratio; IAND, interankle blood pressure difference.

patients with CS with overt PAD to show the implication of mild PAD for stroke prognosis.

We found that large IAND was associated with old age, lower high-density lipoprotein, higher arterial stiffness, and lower ABI. Poor long-term outcome was also independently associated with large IAND in patients with $\mathrm{CS}$ without overt PAD. Moreover, presence of PAD is known to be associated with endothelial dysfunction, ${ }^{16}$ arterial stiffness, ${ }^{17}$ increased left ventricular mass index, ${ }^{18}$ coronary disease ${ }^{19}$ cerebral artery disease,${ }^{20}$ and poor outcome. ${ }^{21}$ In this regard, our study suggests that large IAND may be a surrogate marker of mild PAD in patients with CS without overt PAD, and thus patients with large IAND may have a worse prognosis due to exposure to more risk factors and atherosclerotic burden.

Age is a significant predictor of long-term stroke recurrence and all-cause mortality in patients with CS. ${ }^{4}$ In our study, the prognostic implication of large IAND was age dependent. Elderly patients, but not younger patients, with CS showed a strong association between systolic IAND and stroke outcomes. The interaction between advanced age and difference in the atherosclerotic burden may be one of the determinants of the prognosis of patients with CS.

This study has several limitations. First, there is a possible selection bias owing to the retrospective design. To overcome this, we enrolled consecutive patients with CS. However, depth of cardiac evaluation and presence of hidden cardiac embolic source may affect the results. Second, we measured BP only once. Repeated measurement is recommended to reduce the variation of IAND. Therefore, we repeated BP measurements in patients with ABI $<1.0$ or IAND $\geq 10 \mathrm{~mm} \mathrm{Hg}$ for reliability. Third, not all patients underwent angiographic studies of the lower extremity to diagnose PAD. However, substenotic atherosclerosis may not be visible on lumen imaging ${ }^{2}$ and ABI is recommended for use in clinical practice and research according to guidelines from the American College of Cardiology/American Heart Association.$^{22}$ Fourth, ankle BP may be affected by medial artery calcification or muscle atrophy and stiffening in the legs. Fifth, we did not include other cardiovascular events (coronary revascularisation, hospitalisation for heart failure, etc) in the MACEs. Thus, a caution is required in interpreting the results of this study. Finally, this study was performed in a South Korean population. Studies for different ethnicities are needed.

\section{CONCLUSIONS}

We demonstrated that systolic and diastolic IANDs were significantly associated with the long-term occurrence of MACEs in patients with CS without PAD. In particular, the association between IAND and MACE was only observed in elderly patients. Therefore, we suggest that large IAND may be a useful prognostic marker in CS patients, especially elderly patients with CS.

Contributors MH: conceptualisation, methodology, formal analysis, visualization, resources, project administration, data curation, data validation, writing-original draft. HSN: conceptualisation, methodology, supervision, resources, project administration, data curation, data validation, funding acquisition, writing —review and editing. MB, YDK, EP, and JHH: supervision, data curation, data validation. JC and KS: resources, data validation. HSN is acting as guarantor of the paper.

Funding This research was supported by a grant of the Korea Health Technology R\&D Project through the Korea Health Industry Development Institute (KHIDI), funded by the Ministry of Health \& Welfare, Republic of Korea (grant number : HI19C0481, HC19C0028).

Competing interests None declared.

Patient and public involvement Patients and/or the public were not involved in the design, or conduct, or reporting, or dissemination plans of this research.

Patient consent for publication Not applicable.

Ethics approval The Institutional Review Board of Severance Hospital, Yonsei University Health System, approved this study and waived the need for informed consent because of the retrospective design and observational nature of this study (approval date: 2020-01-16; approval number: 4-2019-1196). 
Provenance and peer review Not commissioned; externally peer reviewed.

Data availability statement Data are available on reasonable request. Deidentified participant data are available on reasonable request.

Open access This is an open access article distributed in accordance with the Creative Commons Attribution Non Commercial (CC BY-NC 4.0) license, which permits others to distribute, remix, adapt, build upon this work non-commercially, and license their derivative works on different terms, provided the original work is properly cited, appropriate credit is given, any changes made indicated, and the use is non-commercial. See: http://creativecommons.org/licenses/by-nc/4.0/.

\section{ORCID iDs}

Minho Han http://orcid.org/0000-0003-2951-0963

Hyo Suk Nam http://orcid.org/0000-0002-4415-3995

\section{REFERENCES}

1 Han M, Kim YD, Park HJ, et al. Prediction of functional outcome using the novel asymmetric middle cerebral artery index in cryptogenic stroke patients. PLoS One 2019;14:e0208918.

2 Li L, Yiin GS, Geraghty OC, et al. Incidence, outcome, risk factors, and long-term prognosis of cryptogenic transient ischaemic attack and ischaemic stroke: a population-based study. Lancet Neurol 2015;14:903-13.

3 Han M, Kim YD, Choi JK, et al. Predicting stroke outcomes using Ankle-brachial index and Inter-Ankle blood pressure difference. J Clin Med 2020;9:1125.

4 Ntaios G, Lip GYH, Vemmos K, et al. Age- and sex-specific analysis of patients with embolic stroke of undetermined source. Neurology 2017:89:532-9.

5 Saver JL. Clinical practice. cryptogenic stroke. N Engl J Med 2016;374:2065-74.

6 Adams HP, Bendixen BH, Kappelle LJ, et al. Classification of subtype of acute ischemic stroke. definitions for use in a multicenter clinical trial. TOAST. trial of ORG 10172 in acute stroke treatment. Stroke 1993;24:35-41.

7 Han M, Kim YD, Park HJ, et al. Brachial-ankle pulse wave velocity for predicting functional outcomes in patients with cryptogenic stroke. $J$ Clin Neurosci 2019;69:214-9.

8 Sheng C-S, Liu M, Zeng W-F, et al. Four-limb blood pressure as predictors of mortality in elderly Chinese. Hypertension 2013;61:1155-60.
9 Chang Y, Kim J, Kim Y-J, et al. Inter-arm blood pressure difference is associated with recurrent stroke in non-cardioembolic stroke patients. Sci Rep 2019;9:12758.

10 Chang Y, Kim J, Kim MH, et al. Interarm blood pressure difference is associated with early neurological deterioration, poor short-term functional outcome, and mortality in noncardioembolic stroke patients. J Clin Neurol 2018;14:555-65.

11 Kim J, Song T-J, Song D, et al. Interarm blood pressure difference and mortality in patients with acute ischemic stroke. Neurology 2013;80:1457-64.

12 Hsu P-C, Lee W-H, Tsai W-C, et al. Usefulness of four-limb blood pressure measurement in prediction of overall and cardiovascular mortality in acute myocardial infarction. Int J Med Sci 2020;17:1300-6.

13 Rodés-Cabau J, Noël M, Marrero A, et al. Atherosclerotic burden findings in young cryptogenic stroke patients with and without a patent foramen ovale. Stroke 2009;40:419-25.

14 Reiner Željko, De Sutter J, Ryden L, et al. Peripheral arterial disease and intermittent claudication in coronary heart disease patients. Int $J$ Cardiol 2021;322:227-32.

15 Han M, Kim YD, Lee I, et al. Low toe-brachial index is associated with stroke outcome despite normal Ankle-brachial index. Front Neurol 2021;12:754258.

16 Sanada H, Higashi Y, Goto C, et al. Vascular function in patients with lower extremity peripheral arterial disease: a comparison of functions in upper and lower extremities. Atherosclerosis 2005;178:179-85.

17 Su H-M, Lin T-H, Hsu P-C, et al. Association of bilateral brachialankle pulse wave velocity difference with peripheral vascular disease and left ventricular mass index. PLoS One 2014;9:e88331.

$18 \mathrm{Su} \mathrm{H}-\mathrm{M}$, Lin T-H, Hsu P-C, et al. Association of interankle systolic blood pressure difference with peripheral vascular disease and left ventricular mass index. Am J Hypertens 2014;27:32-7.

19 Palanca A, Castelblanco E, Betriu Àngels, et al. Subclinical atherosclerosis burden predicts cardiovascular events in individuals with diabetes and chronic kidney disease. Cardiovasc Diabetol 2019;18:93

20 Ratanakorn D, Keandoungchun J, Tegeler $\mathrm{CH}$. Coexistent extra- and intracranial stenosis, cervical atherosclerosis, and abnormal ankle brachial index in acute ischemic stroke. J Stroke Cerebrovasc Dis 2012;21:782-9.

21 Timsit S, Bailly P, Nowak E, et al. Cryptogenic mechanism in ischaemic stroke patients is a predictor of 5-year survival: a population-based study. Eur Stroke J 2016;1:279-87.

22 Writing Committee Members. 2016 AHA/ACC guideline on the management of patients with lower extremity peripheral artery disease: Executive summary. Vasc Med 2017;22:1465-508. 